\title{
Value of a novel diagnostics of rSP13-ELISA toward schistosomiasis elimination in China
}

\author{
ZHOU Xiao-Nong \\ National Institute of Parasitic Diseases, Chinese Center for Disease Control and Prevention, Shanghai 200025, China
}

Received May 4, 2014; accepted May 8, 2014; published online May 15, 2014

Citation: Zhou XN. Value of a novel diagnostics of rSP13-ELISA toward schistosomiasis elimination in China. Sci China Life Sci, 2014, 57: 647-648, doi: $10.1007 / \mathrm{s} 11427-014-4674-6$

Schistosomiasis, caused by infections with Schistosoma japonicum, is one of the human parasitic diseases that remains a public health problem throughout the world's tropical and subtropical regions. With the sustained efforts for six decades, schistosomiasis has been effectively controlled in China. But in the lake region of the schistosomiasis endemic areas, the disease is still a big burden to some specific population, such as fishmen and boatmen, which hinders the local social-economic development. Currently, due to the low infection intensity in population of endemic areas after implementation of the integrated control strategy with multi-interventions [1], such as health education, preventive chemotherapy with praziquantel, mechanization in agriculture with replacement of water buffalo, and snail elimination through environmental modification, sensitive diagnostics still lack for detecting infections and assessing the effectiveness of the control interventions. Recently, The Lancet Infectious Diseases published the findings from Chinese scientists that a single protein marker dramatically increased the sensitivity and specificity of immunodiagnostic testing of schistosome infection in humans [2].

This study was conducted by a research team led by Prof. Pan WeiQing, consisting of researchers from Tongji University School of Medicine, Jiangxi Provincial Institute of Parasitic Diseases, Medical College of Soochow University, and Second Military Medical University. The investigators carried out a genome-wide identification of diagnostic protein markers for schistosome infection and assessed their diagnostic validity in a field study. They predicted secreted proteins (SjSP) of $S$. japonicum was achieved through

email: zhouxn1@ chinacdc.cn screening at genome-wide scale via bioinformatics technology. Briefly, the screening was performed based on the genome sequences along with the corresponding proteomic and transcriptomic datasets [3,4]. The putative secreted proteins were cloned and expressed as glutathione S-transferase fusion proteins (GST-SjSPs). The fusion proteins were arrayed on GSH-immobilized microplates and screened with schistosomiasis patient sera. Only one recombinant protein (SjSP-13), out of 204, reacted to the entire panel of 14 archived samples, although the other 35 yielded seropositive reactions, eight showed strong immunoreactivity. The reactivity of SjSP-13 to 476 serum samples showed higher sensitivity $(90.4 \%)$ and specificity (98.9\%), based on laboratory evaluation [2].

To understand the feasibility of the identified protein marker (SjSP-13) to be used in the national schistosomiasis control program, a field trial was carried out. The field trial was conducted in two villages which located in an endemic area of schistosomiasis in the south-eastern Poyang Lake, Jiangxi Province [2]. Of 1371 enrolled residents, only 74 individuals were identified as being infected by traditional parasitological detection by using Kato-Katz thick smears, while 465 were diagnosed as positive by the SjSP-13-based ELISA kit (rSP13-ELISA). Of the 394 individuals found being egg-negative but rSP13-ELISA-positive, 363 (92.4\%) were confirmed to be positive for schistosome infection by PCR detection of $S$. japonicum $\mathrm{SjR} 2$ retrotransposon (Figure 1). Furthermore, over $70 \%$ of the individuals showed seroconversion and all showed a dramatic decline of the anti-SjSP-13 antibody six months after chemotherapy, further demonstrating these individuals had a schistosome infection. 


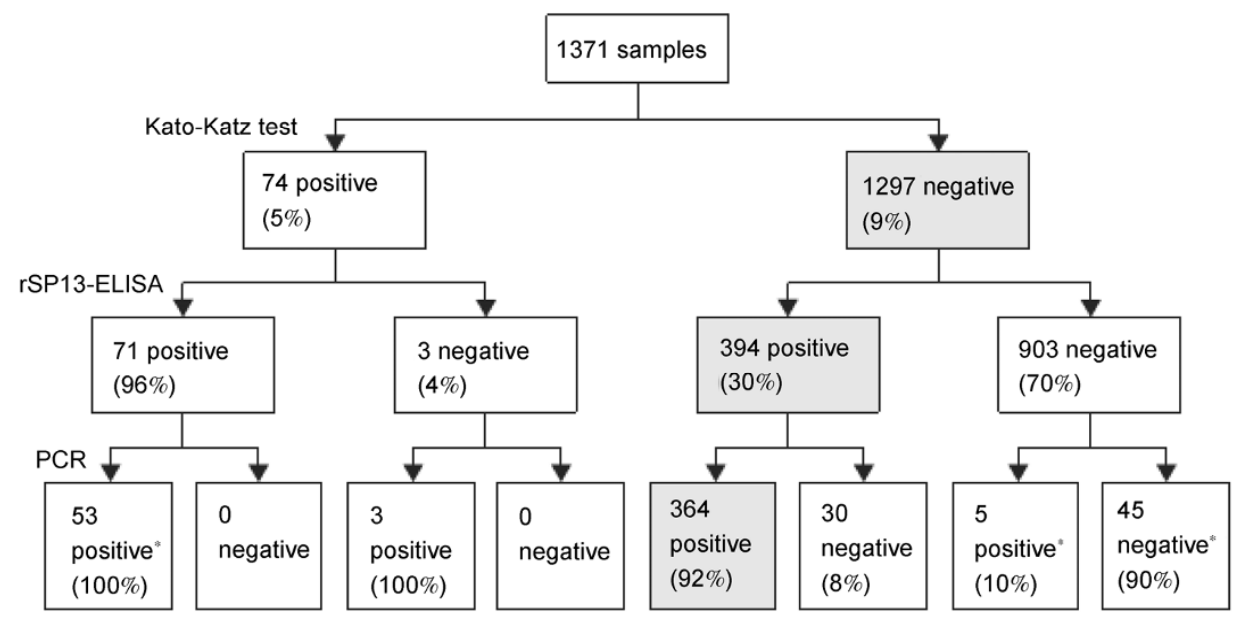

Figure 1 Diagnostic methods used and the outcomes from a field study. Fecal samples from all the participants were examined by Kato-Katz test (27 slides from three samples per individual) and all serum samples were diagnosed by rSP13-ELISA. Samples that were rSP13-ELISA positive were further assayed by PCR for the SjR2 retrotransposon of Schistosoma japonicum. $* 53$ samples diagnosed egg-positive and 50 samples diagnosed both egg and rSP13-ELISA negative were selected for PCR detection.

Currently, the global schistosomiasis control program has made tremendous progress in terms of reducing the schistosomiasis prevalence and morbidity in many endemic countries, particularly after the London Declaration on Neglected Tropical Diseases. With the goal of the disease elimination, low but persistent levels of schistosomiasis transmission require essential changes in many aspects of elimination approaches [5]. For instance, the new tools of diagnosis and surveillance are urgent to be used in the local settings with low-intensity infections of hosts and in the evaluation of intervention effectiveness. The rSP13-ELISA tool developed by Dr. Xu and his colleagues showed substantial advantages over parasitological detection and SEA-ELISA, including adequate sensitivity and specificity needed in low transmission areas [2]. Application of this tool may allow identification of cases with low-intensity infections and targeted treatment.

The National Schistosomiasis Control Program in China (2004-2015) was issued by Ministry of Health in 2004, with the aim of reducing the prevalence of schistosome infection in all endemic counties down to $<5 \%$ by the end of 2008 , and further down to $1 \%$ by the end of 2015 [6]. The success of this program and finally elimination of schistosomiasis in China hinges on the availability of sensitive, simple and affordable diagnostics to be used easily by staff at country level. In the field trial, it has been noticed that only $5 \%$ of the enrolled residents were identified as infected with schistosomes by Kato-Katz thick smears, while $33.9 \%$ were confirmed by virtue of a positive result from rSP13-ELISA [2]. According to the current control program, all infected individuals with $S$. japonicum have to be treated in order to eliminate the transmission. While the rSP13-ELISA approach developed in this study was particularly relevant for mild infections. It performed even better than SEA-ELISA as $89.9 \%$ of mild infections diagnosed correctly by the rSP13-ELISA kit compared to $79.9 \%$ by the SEA-ELISA kit [2]. Therefore, we recommend that further study on cut-off value of the kit is warrant, then it is not so difficult to translate this tool into control program in following three aspects: (i) screening for the treatment of targeted population to achieve the transmission elimination, (ii) screening infected subjects in the surveillance system both in the pre-elimination and post-elimination stages, (iii) providing the certification tool in the elimination of schistosomiasis. Therefore, the research approach to discovery of SjSP-13 protein marker as well as the product of rSP13-ELISA kit could contribute significantly to the elimination of schistosomiasis in China, eventually.

1 Wang LD, Chen HG, Guo JG, Zeng XJ, Hong XL, Xiong JJ, Wu XH, Wang XH, Wang LY, Xia G, Hao Y, Chin DP, Zhou XN. A strategy to control transmission of Schistosoma japonicum in China. N Engl J Med, 2009, 360: 121-128

2 Xu X, Zhang Y, Lin D, Zhang J, Xu J, Liu YM, Hu F, Qing X, Xia C, Pan W. Serodiagnosis of Schistosoma japonicum infection: genomewide identification of a protein marker, and assessment of its diagnostic validity in a field study in China. Lancet Infect Dis, 2014, S1473-3099(14)70067-2

3 Hu W, Yan Q, Shen DK, Liu F, Zhu ZD, Song HD, Xu XR, Wang ZJ, Rong YP, Zeng LC, Wu J, Zhang X, Wang JJ, Xu XN, Wang SY, Fu G, Zhang XL, Wang ZQ, Brindley PJ, McManus DP, Xue CL, Feng Z, Chen Z, Han ZG. Evolutionary and biomedical implications of a Schistosoma japonicum complementary DNA resource. Nat Genet, 2003, 35: 139-147

4 Schistosoma japonicum Genome Sequencing and Functional Analysis Consortium. The Schistosoma japonicum genome reveals features of host-parasite interplay. Nature, 2009, 460: 345-351

5 Zhou XN, Xu J, Chen HG, Wang TP, Huang XB, Lin DD, Wang QZ, Tang L, Guo JG, Wu XH, Feng T, Chen JX, Guo J, Chen SH, Li H, Wu ZD, Peeling RW. Tools to support policy decisions related to treatment strategies and surveillance of schistosomiasis japonica towards elimination. PLoS Negl Trop Dis, 2011, 5: e1408

6 Zheng Q, Vanderslott S, Jiang B, Xu LL, Liu CS, Huo LL, Duan LP, Wu NB, Li SZ, Xia ZG, Wu WP, Hu W, Zhang HB. Research gaps for three main tropical diseases in the People's Republic of China. Infect Dis Poverty, 2013, 2: 15

Open Access This article is distributed under the terms of the Creative Commons Attribution License which permits any use, distribution, and reproduction in any medium, provided the original author(s) and source are credited. 